\title{
A Comparison of Measles Vaccination Regimes in a Stochastic, Spatial SIR Model *
}

\author{
Emily Nguyen ${ }^{\dagger}$ and Amanda Reeder ${ }^{\ddagger}$
}

\author{
Advisor: John Fricks ${ }^{\S}$
}

\begin{abstract}
Measles is a disease that continues to affect millions of people; however, it can now be controlled through vaccination. Using a spatial, stochastic, continuous-time SIR model, we investigate four different vaccination regimes in a "country" of 25 cities. The model was constructed using work by Bjørnstad et al. and May and Anderson as a basis for determining the spatial structure of the country and a set of appropriate parameters [May and Anderson, 1984, Bjørnstad et al., 2002]. We examine the behavior of measles under these vaccination regimes over a period of 20 years with the goal of determining an optimal regime that leads to herd immunity. All data was simulated using an Euler approximation of the continuous-time Markov chain. As vaccination rates increase and begin to induce herd immunity, on average, the same proportion of susceptible people are vaccinated across regimes. However, a closer investigation of the qualitative behavior of these regimes reveals distinct differences among them. Briefly, some regimes maintained a much lower proportion of susceptibles in the population, while others allowed the number of susceptible people in the population to fluctuate significantly. A steady, high vaccination rate across the population eliminated cases and led to herd immunity without such fluctuations.
\end{abstract}

*This research has been presented at the following events: Mathematical Biosciences Institute Capstone Conference (2015), an informal colloquium on student summer research hosted by the Department of Mathematics and Computer Science at Muhlenberg College (2015), Emerging Researchers National Conference in STEAM (2016), and Norfolk State University 2016 Annual Undergraduate Research Symposium. Amanda and Emily both contributed equally to this paper.

${ }_{\dagger} 1949$ Geneva Street, San Jose, CA 95124. Email: emily.lynne@yahoo.com.

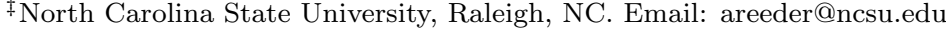

$\S$ School of Mathematical and Scientific Statistics, Arizona State University, Tempe, AZ. Email: john.fricks@asu.edu. 


\section{Introduction}

Although measles can now be prevented through vaccination, outbreaks still occur. The outbreak of measles cases from December 2014 to February 2015 in the United States, which seems to be connected to Disneyland visits [Zipprich et al., 2015], is one example of the threat that measles continues to pose - especially notable in a country in which measles has been essentially eliminated. France began to see an increase in measles cases in 2008 [Freymuth and Vabret, 2011], and Belgium saw outbreaks as recently as 2016 [Grammens et al., 2016]. According to the World Health Organization, measles can result in a few different complications, which especially affect adults older than 20 and children younger than five [WHO, 2015]. These complications can be as minor as an ear infection [WHO, 2015] and as serious as death, although measles is typically not fatal in developed countries [Anderson and May, 1991]. Most fatalities occur among children who do not have access to adequate nutrition. In 2014, there were 114,900 deaths from measles [WHO, 2015]. Even in countries that have mostly eliminated measles, cases may spread quickly in areas where vaccination coverage is limited.

Since there is no antiviral treatment after contracting measles, vaccination is the key to controlling it. However, it is neither practical nor possible to vaccinate every person in a population. In the United States, for example, parents sometimes do not vaccinate children based on an unfounded but widespread belief that vaccinations are connected to autism [Calandrillo, 2004, CDC, 2015b, Flaherty, 2011]. More states are beginning to excuse children from mandatory vaccinations due to parents' "philosophical beliefs," and it is possible to obtain religious exemptions from mandatory vaccinations in almost all states [Calandrillo, 2004]. In countries across the world, people may not have easy access to health care or services, making it difficult for them to obtain vaccinations. Some are not vaccinated simply due to limited access to affordable health care. Others may not take vaccines due to an immune system compromised by other ailments. Meanwhile, measles continues to persist globally. A study of the 2015 outbreak of measles cases in the United States found that approximately 50 to 86 percent of the exposed population were vaccinated [Majumder et al., 2015]; Burkina Faso experienced an epidemic in 2009 that largely affected unvaccinated individuals [Kidd et al., 2012]. While vaccinations are generally required, vaccination regimes are not strictly enforced. Thus, identifying a regime that requires fewer vaccinations and still eliminates cases is an important goal. In this paper, instead of modeling a specific country, we study an idealized system and use historical data to incorporate real-life complexity. This allows us to investigate the spatial aspects of different vaccination regimes on measles.

Because data show that even countries that have mostly eliminated measles may still experience epidemics, herd immunity is needed to prevent them. Herd immunity can be interpreted both quantitatively and qualitatively. Quantitative herd immunity is a partial resistance. The idea is that decreasing the number of source cases and susceptible people will likewise decrease the frequency of the disease. Qualitative herd immunity is a complete resistance; in the words of Fine et al., there is a "threshold number or percentage of [immune people] above which an infection cannot persist" [Fine, 1993]. Both definitions suggest the importance of vaccinating even when a country is not in the midst of an epidemic. For the purposes of our study, we define herd immunity as the state in which measles is endogenously eliminated and in which the percentage of susceptibles (out of the total population) is low, say, $1 \%$ of the total population is susceptible. If measles is not present in the population and if few people are susceptible, it is unlikely that the population will experience an epidemic. We are interested in identifying vaccination regimes that lead to herd immunity.

To study the problem of inducing herd immunity under different vaccination regimes, we will create a stochastic and spatial susceptible-infected-recovered (SIR) model inspired by previous theoretical and empirical work and test it under various regimes. There are a number of excellent foundational resources for stochastic models in disease dynamics [Allen et al., 2008], [Allen, 2010], [Britton, 2010], [Rock et al., 2014]. Moreover, there is already a considerable body of work on stochastic SIR models, which can be used to model measles as well as other diseases. Focused on theoretical results, Ball and Neal consider stochastic SIR models of "non-locally-large populations" [Ball and Neal, 2002]. As these populations grow in size, they retain the same structure at the local level; moreover, "two levels of mixing" are considered [Ball and Neal, 2002]. Ball and Lyne studied vaccination regimes in a SIRS model of a population divided into $m$ households of the same size, but note that this model is a general version of the SIR model and several others [Ball and Lyne, 2002]. As in Ball and Neal, individuals have the opportunity to make global contacts (between households) and local contacts (within households) [Ball and Lyne, 2002]. Verdasca et al. examined stochastic SIR and SEIR models, in which populations are represented by networks and each node on the network represents an individual [Verdasca et al., 2005]. Instead of considering a population composed of, for example, households, Verdasca et al. consider a small world network, which aims to mimic a "social network" [Verdasca et al., 2005]. 
Although Verdasca et al. modeled measles [Verdasca et al., 2005], a good deal of work has also been done on stochastic models that focus more specifically on measles. Benjamin Bolker and Bryan Grenfell explored the effect of age and spatial structure on the persistence and dynamics of measles epidemics, finding that spatial models can capture the persistence and triennial behavior of the disease [Bolker and Grenfell, 1995]. Grenfell et al. studied several scenarios in the pre-vaccine era and focused on the effect of spatial structure on measles over a short period of time. In particular, they used wavelet phase analysis to study models that were fit to pre-vaccine epidemics and then analyzed the traveling waves of measles [Grenfell et al., 2001]. Caudron et al. fit data on measles in some subendemic, secluded populations (Bornholm, the Faroe Islands, and parts of Iceland) using a time series-susceptible-infected-recovered (TSIR) model. They found that they were able to predict how large future epidemics would be "conditioned on the first time-step" [Caudron et al., 2015]. Dalziel et al. fit data on measles in England, Wales, and the US during the pre-vaccine era to a TSIR model. They were able to model "persistent chaotic epidemics" in the US from "small shifts in seasonal transmission patterns" [Dalziel et al., 2016].

Also using a TSIR model, Bjørnstad et al. analyzed data on measles in England and Wales before vaccination was a possibility. Their model captured both endemic cycles and episodic outbreaks of measles and included a stochastic element in the disease transmission and random immigration [Bjørnstad et al., 2002]. Although deterministic, May and Anderson have considered a spatial model in which a large city is surrounded by several smaller cities, and obtained an "optimal" vaccination regime that differed from the ideal vaccination regime for a homogeneously mixing population [May and Anderson, 1984].

For the first time, we study the behavior of different vaccination regimes in a spatial, stochastic, continuoustime SIR model with the goal of identifying whether one regime is "optimal." In other words, we pose May and Anderson's questions about optimal vaccination regimes in a population composed of various subpopulations, but employ an approach more similar to that of Bjørnstad et al.'s stochastic model. Because measles is such a contagious disease, spatial structure and stochasticity are particularly important. The spatial structure of a population not only affects the spread of the disease, but also the efficacy of vaccination regimes. The stochasticity of our model captures any randomness that may affect the behavior of measles in a population. The effects of random population movement are particularly salient because of the easy transmissibility of measles: mere contact with an infected person or even with something she has touched puts a susceptible person at risk of contracting the disease. The stochastic model can also show the fadeout phenomenon [Ferrari et al., 2013], which will be useful for simulating smaller cities that will not have constant measles incidence. Finally, we rely heavily on Bjørnstad et al.'s analysis of empirical data on measles to determine the specifics of our model, such as city size and other parameters. Our final model is in some ways a hybrid of May and Anderson's model and Bjørnstad et al.'s study.

We examine the behavior of four different vaccination regimes in this spatial, stochastic, continuous-time SIR model. Each of these regimes posits a different scheme for distributing vaccinations. Does blanket mass coverage work best? Is there a better time of year to vaccinate? Should vaccinations only be distributed to certain sectors of the population? Is it enough to vaccinate only when measles is present in the immediate area? Our goal was to determine which, if any, of these regimes leads to herd immunity. The preliminary results of our work suggest that vaccinating at a constant rate throughout the year is most effective at both eliminating cases and keeping the proportion of susceptibles low.

We first give an overview of our model and how we represent a "country" of 25 cities. This begins with a closer look at the model for one city and how it is represented using continuous-time Markov chains (§2.1). We then discuss the complete model, focusing on spatial structure, migration, and transmission ( $\$ 2.2)$. Following is a discussion of each of the vaccination regimes we tested in the model ( $§ 3)$. Next, we discuss how to compare the results of the vaccination regimes and make those comparisons (§4). We conclude by summarizing the optimality of the regimes and make a few observations about how that optimality was determined $(\S 5)$.

\section{Overview of the Model}

We model a "country" of 25 cities that are placed on a 5-by-5 grid, as shown in Figure 2. In this country, we distinguish three types of cities, which differ in size and their locations on the grid. The largest cities are located at the center of the grid, and cities become smaller as one moves towards the edges of the country. This layout is similar to a model considered by May and Anderson [May and Anderson, 1984], and a more detailed overview of this model will be given later. The simple SIR model with vaccination serves as a building block for this more extensive spatial model, and we will first examine a component of this model for one city. 


\subsection{SIR Model with Vaccination: Modeling One City}

Each city in the country is modeled using a variation of the SIR model. Following the traditional SIR model, each individual in a city is classified as susceptible, infected, or recovered. A detailed description of assumptions made for SIR models can be found in [Rock et al., 2014]. However, we highlight several assumptions here:

- A recovered person is defined as someone who has either been vaccinated or who has had the disease and is no longer contagious. This assumption is made because, in both cases, the person no longer affects the infection process.

- At any point in time, a person can enter the susceptible compartment, the infected compartment, or the recovered compartment.

- We assume homogeneous mixing of people within each compartment. Essentially, all individuals can come into contact with one another, regardless of age, location, etc. within a city. We make this assumption for simplicity and will add other types of complexity in our final model.

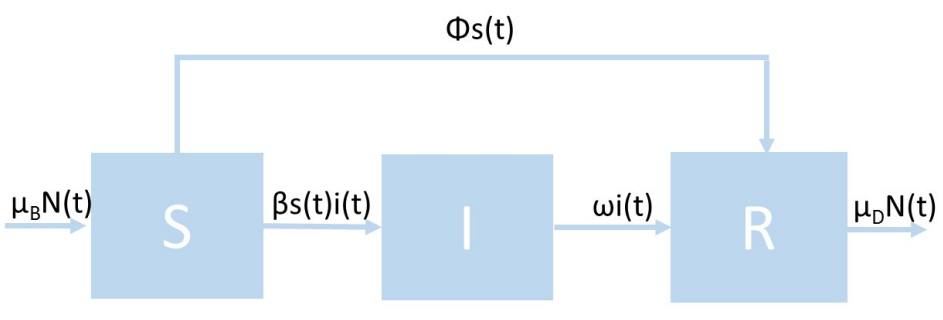

Figure 1: A flow diagram of a SIR with vaccination model. This diagram shows how people can move amongst the compartments within one city (we leave out movement from another city). The arrows pointing into the compartments represent a person entering a compartment, and the arrows pointing out represent a person leaving the compartment.

Figure 1 demonstrates the types of movement that can occur among the three compartments in this model.

In a model of one city with no immigration, there are five "events" or "reactions" that may occur: a birth, an infection, a vaccination, a recovery, or a death. (In our final model, we allow movement between cities, or "emigration.") When one of these events happens, the value of at least one compartment will change. If a person is born, one person enters S; likewise, if a person dies, one person leaves R. If a person becomes infected, one person leaves $\mathrm{S}$ and one - in fact, the same - person enters I. When a person is vaccinated, one person leaves $\mathrm{S}$ and enters $\mathrm{R}$ (representing that the person has immunity). If a person recovers, one person leaves I and one enters R. We can represent these events in a stoichiometric matrix, where each row represents the change in $\mathrm{S}$, $\mathrm{I}$, and $\mathrm{R}$ and each column represents a different event. For one city, this matrix, which we will call $A$, looks as follows:

$$
A=\left(\begin{array}{ccccc}
1 & -1 & -1 & 0 & 0 \\
0 & 1 & 0 & -1 & 0 \\
0 & 0 & 1 & 1 & -1
\end{array}\right) .
$$

The first row represents $\mathrm{S}$, the second I, and the third R; the columns represent a birth, infection, vaccination, recovery, and death respectively.

We must now determine the rates at which these events happen - that is, the rates at which people move among the compartments. These rates are all conditioned on the state of the system at the current time, $t$, and have time units of two weeks, which is about the "characteristic time scale" of measles [Bjørnstad et al., 2002]. In terms of the compartments, this represents the time it takes for someone to move from the $\mathrm{S}$ compartment through the I compartment to the R compartment.

Let $N(t)$ be the total population, $S(t)$ the number of people in compartment $\mathrm{S}, I(t)$ the number of people in compartment I, and $R(t)$ the number of people in compartment $\mathrm{R}$ at time $t$. (Since we concentrate on modeling one city in this section, we eliminate the subscript $(i, j)$ that will eventually identify the city's position in the country.) Then we have the following rates:

- $\mu_{B} N(t)$ : Birth rate (the rate at which people enter $\mathrm{S}$ ),

- $\beta S(t) I(t)$ : Infection rate (the rate at which people move from $\mathrm{S}$ to I), 
- $\phi S(t)$ : Vaccination rate (the rate at which people move from $\mathrm{S}$ to $\mathrm{R}$ ),

- $\omega I(t)$ : Recovery rate (the rate at which people move from I to $\mathrm{R}$ ), and

- $\mu_{D} N(t)$ : Death rate (the rate at which people leave $\mathrm{R}$ ).

Each of these rates are scaled by population size, each in a particular way. Since the number of births and deaths depends on the number of people in the total population $(\mathrm{S}+\mathrm{I}+\mathrm{R})$, the birth rate $\left(\mu_{B}\right)$ and the death rate $\left(\mu_{D}\right)$ are multiplied by the population of each city. Similarly, the number of people who become infected depends on the number of susceptibles and the number of infected. $\beta S(t) I(t)$ is the force of infection and is multiplied by the number of susceptibles and the number of infecteds; thus, $\beta S(t) I(t)$ gives the rate at which a susceptible person becomes infected after coming into contact with an infected person. The vaccination rate $(\phi)$ depends on the number of susceptibles, as only susceptible people should be vaccinated. Finally, the recovery rate $(\omega)$ is multiplied by $I(t)$ since only infected people will move from the infected compartment to the recovered compartment. The unit of time for each of these rates is people per two weeks. For instance, the infection rate is the average number of people that become infected every two weeks.

The model can be simulated in $\mathrm{R}$ using continuous-time Markov chains. Although we could easily express a deterministic version of our model using differential equations, Markov chains allow us to incorporate randomness in our model. That is, each of these reactions - births, deaths, infections, vaccinations, etc. - is represented by

a set of stochastic terms. Given the initial values of the compartments, we can easily determine the values of the compartments at time $t$ :

$$
\left(\begin{array}{l}
S(t) \\
I(t) \\
R(t)
\end{array}\right)=\left(\begin{array}{c}
S(0) \\
I(0) \\
R(0)
\end{array}\right)+\underbrace{\left(\begin{array}{ccccc}
1 & -1 & -1 & 0 & 0 \\
0 & 1 & 0 & -1 & 0 \\
0 & 0 & 1 & 1 & -1
\end{array}\right)}_{A}\left(\begin{array}{c}
Y_{1}\left(\int_{0}^{t} \mu_{B} N(s) d s\right) \\
Y_{2}\left(\int_{0}^{t} \beta S(s) I(s) d s\right) \\
Y_{3}\left(\int_{0}^{t} \phi S(s) d s\right) \\
Y_{4}\left(\int_{0}^{t} \omega I(s) d s\right) \\
Y_{5}\left(\int_{0}^{t} \mu_{D} N(s) d s\right)
\end{array}\right)
$$

Recall that $A$ is a stoichiometric matrix, each column of which represents a different reaction. $Y_{1}, Y_{2}, \ldots, Y_{5}$ are time-changed unit one Poisson processes - one for each of the five events or reactions. A more detailed description of how the model is simulated can be found in appendix A.

\subsection{Complete Model}

The SIR model with vaccination and the rates we have discussed so far model measles in one population. May and Anderson studied a model that serves as a template for modeling the dynamics of measles in a country that is composed of multiple cities [May and Anderson, 1984]. In particular, they studied a population divided into $n$ groups, each of which mixes homogeneously (with heterogeneous mixing among the groups) [May and Anderson, 1984]. It is also worth noting that they use a standard set of differential equations to model the disease [May and Anderson, 1984]. In our model, we differ by using a stochastic model. Bjørnstad et al. analyzed data on measles in England and Wales during the pre-vaccination era. They used a time-series SIR model to capture both endemic cycles and episodic outbreaks of measles and included a stochastic element in the disease transmission and random immigration. The parameters are estimated using time series data on reported cases as well as reconstructed susceptibles from a set of cities in England and Wales [Bjørnstad et al., 2002]. May and Anderson's work thus provided the framework for our model, while Bjørnstad et al.'s data analysis provided a tractable set of parameters.

A brief list of assumptions for our spatial model is given below, and more detailed explanations of these assumptions can be found later on in this section.

- A country is composed of multiple cities [May and Anderson, 1984].

- The population is broken up into $n$ groups (or cities) [May and Anderson, 1984].

- Each city mixes homogeneously with heterogeneous mixing between cities [May and Anderson, 1984]. This is also reflected in Bjørnstad et al.'s data analysis, which observes different immigration rates for different cities [Bjørnstad et al., 2002]. 
- The largest city is centered in the middle of the country, and the population becomes less dense as one moves toward the edge of the country [May and Anderson, 1984]. City sizes are taken from Bjørnstad et al.'s data analysis [Bjørnstad et al., 2002].

- Only infected individuals may emigrate because they are the only individuals that have the ability to significantly alter the dynamics of measles in a population.

- Infected individuals may only emigrate in the horizontal and vertical directions. We add this assumption for simplicity. The exception is the capital, which receives infected immigrants from outside the country. This immigration rate of infected individuals from outside the country reflects a phenomenon seen in countries in which measles has mostly disappeared, such as the United States [CDC, 2015a].

- The rate at which infected individuals may emigrate depends on the type of city from which they are emigrating. This is due to the fact that the immigration rate depends on the size of the city [Bjørnstad et al., 2002].

- The force of infection varies over time since measles tends to spread more quickly when children are in school [Bjørnstad et al., 2002].

Our final model investigates the dynamics of measles in a country of 25 cities. Each city has its own set of susceptibles, infecteds, and recovereds, denoted by $S_{(i, j)}(t), I_{(i, j)}(t)$, and $R_{(i, j)}(t)$ respectively. The cities can be classified as towns, regional centers, or the capital, and they have initial populations of 10,000,500,000, and $5,000,000$ respectively (Figure 2). These population sizes reflect the sizes of cities in England and Wales before a vaccine existed [Bjørnstad et al., 2002]. Each of these cities is placed on a 5-by-5 grid with the capital at the center (Figure 2). The four regional centers surround the capital, and the rest are towns. Mimicking May and Anderson's model, we divide the population into subgroups (cities) and arrange cities so that the most populated city is at the center. We also follow their lead by assuming homogeneous mixing within each city and heterogeneous mixing among different cities.

It is also important to note that we ignore the immigration of susceptibles and recovereds. The movement of a few susceptibles or recovereds is not significant enough to drastically change the dynamics of measles in a city. Immigration, on the other hand, especially of an infected person into a population high in susceptibles, can spark an epidemic. Thus, only infected people can move to neighboring cities, and for simplicity, we only allow vertical and horizontal immigration. The capital, regional centers, and the four towns diagonal to the capital all connect with four other cities. The towns on the perimeter of the grid connect with three, and the corner towns of the grid only connect with two. This structure represents more movement near big cities, with the amount of movement decreasing as one moves away from the capital.

As we discussed in the previous section, each city in the country can be represented by an SIR model with vaccination, which accounts for births, deaths, infections, recoveries, and vaccinations within each city.

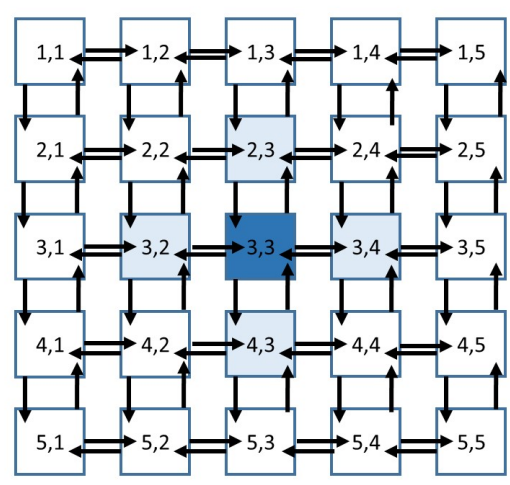

Figure 2: This depicts the layout of the "country" that is being modeled. Regional centers are colored light blue, the capital is dark blue, and the remaining cities are towns. The numbers inside of the squares represent the position of the city on the grid. Arrows indicate possible immigration patterns of infected individuals. For instance, an infectious individual from the capital can move from node $(3,3)$ to node $(3,2),(3,4),(2,3)$, or $(4,3)$. Note that infective individuals can also immigrate from outside the "country" into the capital.

In the final model, however, we have one more event to consider: emigration/immigration of infected people. Each type of city has a different emigration rate: The capital has the highest emigration rate $\left(\delta_{C}\right)$, followed 
by the regional centers $\left(\delta_{R}\right)$, and then the towns $\left(\delta_{T}\right)$. Because we only allow the emigration of infected people, the rate at which people leave a city depends on the number of infected people in the city. In a town, for example, $\delta_{T} I_{(i, j)}(t)$ is the rate at which infected individuals emigrate from city $(i, j)$ to another city in the country. The capital also has one additional immigration rate $\left(\delta_{O}\right)$ to account for immigration from outside the country. This rate was necessary in order to keep measles from disappearing from the entire country [Bolker and Grenfell, 1995, and references therein]. The outside immigration rate is actually not as arbitrary as it might seem: now, any case reported in the United States either can be traced to a case contracted in another country or was itself contracted in another country [CDC, 2015a].

Finally, we want to make a special note about the force of the infection, $\beta$. The input value $\beta$ varies throughout the year; in particular, there is a higher level of infection while children are at school - and thus more likely to interact with one another-than during summer [Bjørnstad et al., 2002]. Using Olsen et al., we estimate an equation for the force of infection:

$$
\beta_{t}=\bar{\beta}-0.4 \bar{\beta} \sin \left(\frac{\pi}{13}(t+\theta)\right),
$$

where $\bar{\beta}$ is calculated using $\log (\bar{\beta})=3.64-1.02 \log (N)$ [Bjørnstad et al., 2002, Olsen and Schaffer, 1990]. Because $\bar{\beta}$ depends on $N$ (the total population of the city), we have three different $\beta$ functions. The capital has the lowest infection rate and the towns have the highest, reflecting the fact that measles spreads most easily when there is a concentration of susceptible individuals in the population. This function also approximates the fluctuations in the rate by peaking at the end of January and reaching a low point at the end of August. A table of parameters and variables can be found in Table 1, and a more detailed explanation of the parameters can be found in appendix B.

\begin{tabular}{ccc}
\hline Variable/Parameter & Description & Units \\
\hline \hline$N_{T}=10,000$ & Total initial population of a town & people \\
$N_{R}=500,000$ & Total initial population of a regional center & people \\
$N_{C}=5,000,000$ & Total initial population of capital & people \\
$S / N=0.008$ & Initial proportion of susceptibles in each city & susceptibles/total people in city \\
$I / N=0.007$ & Initial proportion of infected people in each city & infected $/$ total people in city \\
$\mu_{B}=\log (1.03) / 26$ & Birth rate for each city in country & avg. no. births $/ 2$ wks \\
$\mu_{D}=.00025$ & Death rate for each city in country & avg. no. deaths $/ 2$ wks \\
$\delta_{T}=.2 / \sqrt{10000}$ & Emigration rate of a town & avg. no. emigrations $/ 2$ wks \\
$\delta_{R}=4 / \sqrt{500000}$ & Emigration rate of a regional center & avg. no. emigrations $/ 2$ wks \\
$\delta_{C}=20 / \sqrt{5000000}$ & Emigration rate of capital & avg. no. emigrations $/ 2$ wks \\
$\delta_{O}=2$ & Immigration rate to capital from outside country & avg. no. immigrations $/ 2$ wks \\
$\beta_{C}(t)$ & Infection rate in capital at time $t$ & per infected indiv. at time $t$ \\
$\beta_{R}(t)$ & Infection rate in regional centers at time $t$ & per infected indiv. at time $t$ \\
$\beta_{T}(t)$ & Infection rate in towns at time $t$ & per infected indiv. at time $t$ \\
$t$ & Time & 2 weeks \\
$S_{(i, j)}(t)$ & No. of susceptibles in city $(i, j)$ at time $t$ & people \\
$I_{(i, j)}(t)$ & No. of infecteds in city $(i, j)$ at time $t$ & people \\
$R_{(i, j)(t)}(t)$ & No. of recovereds in city $(i, j)$ at time $t$ & people \\
$N_{(i, j)}(t)$ & No. of total people in city $(i, j)$ at time $t$ & people \\
\hline
\end{tabular}

Table 1: The parameters and variables in the model and the units for each. A more detailed discussion of how we determined these parameters can be found in appendix B.

Using continuous-time Markov chains, we can simulate the values of the compartments of each city in our final model. We use a method similar to that described in $\S 2.1$. For any town or regional center $(i, j)$, we use the stoichiometric matrix mentioned before, but add the events of emigration and immigration. This matrix, which looks as follows, represents all possible events that may occur in the model:

$$
A=\left(\begin{array}{ccccccc}
1 & -1 & -1 & 0 & 0 & 0 & 0 \\
0 & 1 & 0 & -1 & 0 & -1 & 1 \\
0 & 0 & 1 & 1 & -1 & 0 & 0
\end{array}\right)
$$


The second-to-last column of the stoichiometric matrix represents the emigration of an infected person (a person that leaves city $(i, j)$ for another city). The last column represents the immigration of an infected person into city $(i, j)$.

Thus, the values of the compartments $\mathrm{S}, \mathrm{I}$, and $\mathrm{R}$ in city $(i, j)$ can be determined as follows. Let $\nu$ be the neighborhood of city $(i, j)$, where $\nu$ is the set of all cities from which infected individuals may emigrate to city $(i, j)$. In simpler terms, $\nu$ is the set of all cities that connect to city $(i, j)$. We also note that $\beta_{(i, j)}(t)=\beta_{T}(t)$ if city $(i, j)$ is a town and $\beta_{(i, j)}(t)=\beta_{R}(t)$ if $(i, j)$ is a regional center. Similarly, $\delta_{(i, j)}=\delta_{R}$ if $(i, j)$ is a regional center and $\delta_{(i, j)}=\delta_{T}$ if $(i, j)$ is a town. Then,

$$
\left(\begin{array}{c}
S_{(i, j)}(t) \\
I_{(i, j)}(t) \\
R_{(i, j)}(t)
\end{array}\right)=\left(\begin{array}{c}
S_{(i, j)}(0) \\
I_{(i, j)}(0) \\
R_{(i, j)}(0)
\end{array}\right)+\underbrace{\left(\begin{array}{ccccccc}
1 & -1 & -1 & 0 & 0 & 0 & 0 \\
0 & 1 & 0 & -1 & 0 & -1 & 1 \\
0 & 0 & 1 & 1 & -1 & 0 & 0
\end{array}\right)}_{A}\left(\begin{array}{c}
Y_{1}\left(\int_{0}^{t} \mu_{B} N_{(i, j)}(s) d s\right) \\
Y_{2}\left(\int_{0}^{t} \beta_{(i, j)} S_{(i, j)}(s) I_{(i, j)}(s) d s\right) \\
Y_{3}\left(\int_{0}^{t} \phi S_{(i, j)}(s) d s\right) \\
Y_{4}\left(\int_{0}^{t} \omega I_{(i, j)}(s) d s\right) \\
Y_{5}\left(\int_{0}^{t} \mu_{D} N_{(i, j)}(s) d s\right) \\
Y_{6}\left(\int_{0}^{t} \delta_{(i, j)} I_{(i, j)}(s) d s\right) \\
\sum_{\forall(m, n) \in \nu} Y_{7}\left(\int_{0}^{t} \delta_{(m, n)} I_{(m, n)}(s) d s\right)
\end{array}\right) .
$$

Because the capital has one additional event (the immigration of infected people from outside the country), we must adjust the stoichiometric matrix and add one time-changed unit one Poisson process. We add one column to the stoichiometric matrix $A$ to represent the immigration of infected people. Again, let $\nu$ be the neighborhood of cities that connect to the capital. Then, at time $t$, the values of the compartments $\mathrm{S}, \mathrm{I}$ and $\mathrm{R}$ are as follows:

$$
\left(\begin{array}{c}
S_{(3,3)}(t) \\
I_{(3,3)}(t) \\
R_{(3,3)}(t)
\end{array}\right)=\left(\begin{array}{c}
S_{(3,3)}(0) \\
I_{(3,3)}(0) \\
R_{(3,3)}(0)
\end{array}\right)+\underbrace{\left(\begin{array}{cccccccc}
1 & -1 & -1 & 0 & 0 & 0 & 0 & 0 \\
0 & 1 & 0 & -1 & 0 & -1 & 1 & 1 \\
0 & 0 & 1 & 1 & -1 & 0 & 0 & 0
\end{array}\right)}_{A}\left(\begin{array}{c}
Y_{1}\left(\int_{0}^{t} \mu_{B} N_{(3,3)}(s) d s\right) \\
Y_{2}\left(\int_{0}^{t} \beta_{C} S_{(3,3)}(s) I_{(3,3)}(s) d s\right) \\
Y_{3}\left(\int_{0}^{t} \phi S_{(3,3)}(s) d s\right) \\
Y_{4}\left(\int_{0}^{t} \omega I_{(3,3)}(s) d s\right) \\
Y_{5}\left(\int_{0}^{t} \mu_{D} N_{(3,3)}(s) d s\right) \\
Y_{6}\left(\int_{0}^{t} \delta_{(3,3)} I_{(3,3)}(s) d s\right) \\
\sum_{\forall(m, n) \in \nu} Y_{7}\left(\int_{0}^{t} \delta_{(m, n)} I_{(m, n)}(s) d s\right) \\
Y_{8}\left(\int_{0}^{t} \delta_{O} d s\right)
\end{array}\right) .
$$

The stoichiometric matrix for our final model was 75x206 - 75 total compartments (three for each of the 25 cities) and 206 events to monitor. The model, including the vaccination regimes, was simulated in $\mathrm{R}$ using continuous-time Markov chains. Our first approach for these simulations was to use the Gillespie SSA [Gillespie, 1977] to calculate the number of people in each compartment. This exact method was computationally expensive due to the size of the model, so we used an Euler approximation of continuous-time Markov chains to estimate the values instead. More details on both methods can be found in appendix A.

\section{$3 \quad$ Vaccination Regimes}

There are many possible strategies for vaccinating a population, and each of the regimes investigates a different aspect or technique of distribution. We tested four different regimes in the model: two static and two dynamic. For each of these regimes, we conducted a parameter sweep to examine the behavior of the model under different vaccination levels. Note that $\phi$ denotes the vaccination parameter.

\section{Static Constant Regime}

The static constant regime calls for an essentially equitable distribution of vaccinations. Under this regime, the same proportion of susceptibles is vaccinated in each city. In other words, regardless of whether a city is classified as the capital, a regional center or a town, the same proportion of that city's population is vaccinated. Moreover, this rate stays constant throughout the year. This blanket vaccination regime is not the most practical: the farther away people are from a large city, the harder it might be to be vaccinated. This is, however, a simple and straightforward approach to reach herd immunity. We tested eight different values for $\phi: 0.02,0.04$, 
$0.06,0.08,0.1,0.12,0.14$ and 0.16 .

\section{Static City Regime}

The static city regime vaccinates a different proportion of susceptibles in each class of city: that is, there are different vaccination rates for the capital $\left(\phi_{C}\right)$, the regional centers $\left(\phi_{R}\right)$, and the towns $\left(\phi_{T}\right)$. Note that these rates remain constant throughout the year. The idea for this regime comes from May and Anderson, who posited that vaccinating a larger proportion of people in the big city (in our case, the capital) would more effectively control measles than vaccinating the same proportion of people across all cities [May and Anderson, 1984]. This regime is plausible. Denser populations have more access to health care than sparse populations; because of this, it is more challenging to vaccinate uniformly across a country. Furthermore, measles spreads faster in denser populations, so more vaccination in these areas could potentially counteract this effect. Finally, examining this regime allows us to test in a stochastic setting the hypothesis of May and Anderson, who found that vaccinating only the densest population minimized the number of cases and the number of vaccinations for the country as a whole [May and Anderson, 1984].

We tested three sets of parameters for this regime (Table 2). The first tests the behavior of measles when only one type of city is vaccinated (only towns, only regional centers, or only the capital). The next two sets test higher vaccination levels in the capital and lower vaccination rates in the regional centers and towns. The vaccination rate in the regional centers is always half the rate in the capital, while the rate in the towns is only a quarter of the capital rate. These last two sets of parameters realistically simulate vaccination distribution, which may not always be equitable due to access to health care and other variables.

\begin{tabular}{cccc}
\hline Regime & $\phi_{T}$ & $\phi_{R}$ & $\phi_{C}$ \\
\hline \hline 1 & 0.16 & 0 & 0 \\
2 & 0 & 0.16 & 0 \\
3 & 0 & 0 & 0.16 \\
\hline 4 & 0 & 0.08 & 0.16 \\
5 & 0.04 & 0.08 & 0.16 \\
\hline 6 & 0 & 0 & 0.12 \\
7 & 0 & 0.06 & 0.12 \\
8 & 0.03 & 0.06 & 0.12 \\
\hline
\end{tabular}

Table 2: A table of the eight static city regimes with specific vaccination rates. This regime, influenced by May and Anderson's work [May and Anderson, 1984], vaccinates different proportions of susceptibles in each type of city. Although vaccination rates differ across cities, vaccination rates remain constant.

\section{Dynamic Time Regime}

The dynamic time regime vaccinates a different proportion of susceptibles throughout the year. It has been shown that the force of infection varies throughout the year; in particular, there is a higher level of infection while children are at school - and thus more likely to interact with one another - than in summer [Bjørnstad et al., 2002]. Since the infection rate varies through time, peaking in the fall, we hypothesize that increasing vaccinations a few months before measles peaks might help to control the spread of the disease. The function for $\phi(t)$ is as follows:

$$
\phi(t)=0.002 \sin \left(\frac{\pi}{13}(t+8)\right)+a .
$$

The amplitude of this function was determined by finding $\hat{\beta}$, the mean sample force of infection. $0.4 \hat{\beta}$ is approximately equal to the amplitude of the $\beta$ function. In order to find $\hat{\beta}$, we ran the model for 20 years without vaccination and collected the infection rates, $\beta s i \Delta$. To determine $\hat{\beta}$, we simply divided by $s, i$, and $\Delta=0.001$. On average, $\hat{\beta}=0.002$. This function is also shifted so that it peaks around $t=8$. Since we are working in time units of 2 weeks, this corresponds to a peak vaccination rate in April - several months before school starts and the infection rate begins to rise. We ran 8 tests for this regime, varying the value of $a$ for $a=$ $0.02,0.04,0.06,0.08,0.1,0.12,0.14$, and 0.16 .

\section{Dynamic Infected Regime}

The dynamic infected regime vaccinates a different proportion of susceptibles depending on the number of infected people in the city. That is, if there is at least one infected person in the city, the vaccination rate 
increases. The rationale behind this regime is that vaccinating at a higher rate once there is an infected person in the city will prevent measles from spreading further. This regime is also one that can be "self-imposed" by a population. That is, as people begin to forget about the dangers of measles, they begin vaccinating at a lower rate; once cases appear, they start to vaccinate more. The vaccination rate for this regime thus looks as follows:

$$
\phi_{1} s+\phi_{2} s 1_{\{i \neq 0\}} .
$$

Here, 1 is an indicator function that determines whether the infected compartment is at 0 or not. If the indicator returns a value of true, the vaccination rate is $\phi_{1} s+\phi_{2} s$; otherwise, it remains at the base vaccination rate of $\phi_{1} s$. We keep $\phi_{1}$ constant at 0.02 and run tests for $\phi_{2}=0.02,0.04,0.06,0.08,0.1,0.12,0.14$, and 0.16 .

\section{Results}

In this section, we will examine the behavior of the model under the four different vaccination regimes. After looking at the average proportion vaccinated versus the average proportion infected in each of the regimes, we will focus on a set of regimes that approach herd immunity and the qualitative differences observed there. We will then briefly discuss some observed similarities between the static constant and dynamic time regimes. The static city regime also deserves some special attention since it can be contrasted with the result of May and Anderson. We conclude this section with a short discussion of the behavior of the vaccination regimes at lower levels of vaccination. Before taking a look at the behavior of these regimes, we consider the behavior of the

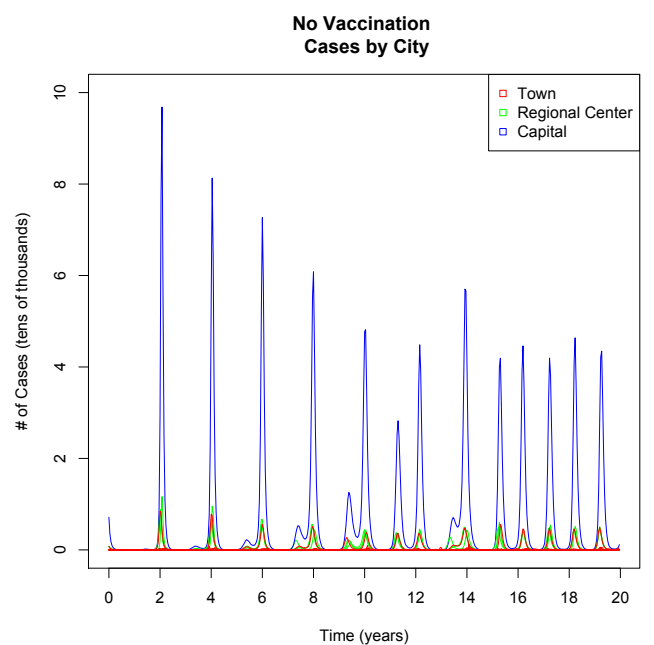

Figure 3: The number of cases in a model without vaccination, with each type of city graphed in a different color. Note the oscillating behavior of the cases in this figure and Figure 4.
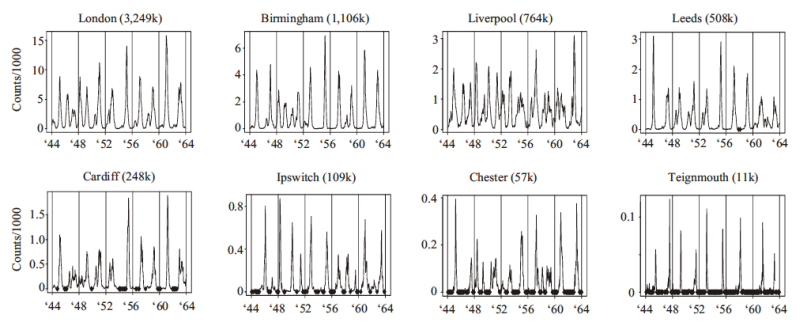

model without vaccination. Figure 3 shows that the number of cases is quite high, reaching nearly 100,000 in the capital. Moreover, we see the number of cases spike and fall about every two years, although epidemics seem to become slightly more frequent towards the end of the 20-year period. The graph of cases in our model is encouragingly similar to the graph in Figure 4, which shows the behavior of measles in English and Welsh cities post-WWII. (This graph was taken from Bjørnstad et al.) In both graphs, we can see the number of cases consistently rise and fall over a 20-year period.

\subsection{Vaccinations vs. Cases}

In order to determine whether one regime was more effective than the others, we first looked at the mean proportion vaccinated every year versus the mean proportion infected each year for each of the tests we ran. 


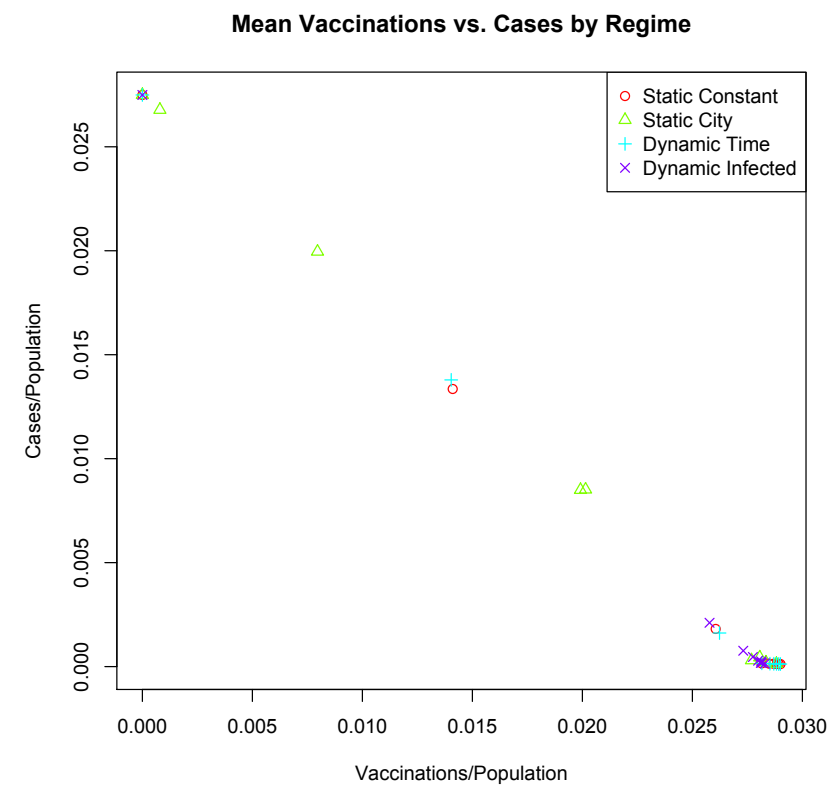

Figure 5: The average number of vaccinations and cases in each test of each regime. The means are calculated by taking the number of vaccinations and cases per year and dividing by total population; these proportions are then averaged to get the mean number of vaccinations and cases over the 20-year period. Each point on the graph represents a different test of a different regime. For example, the green triangle at the top left represents one test we ran for the static city regime.

These means were calculated by taking the total number of vaccinations and cases that occurred in a year and dividing by the total population in the country. This gives the proportion of people vaccinated and the proportion of people infected in a given year. To get the mean number of vaccinations and cases over the 20-year period, we averaged the proportions.

Since it is difficult to make fair comparisons using the rates of vaccination (i.e., $\phi$ ) given the other differences in the model, we use the means as proxies. If one regime significantly reduces the proportion infected while vaccinating the same proportion as the other regimes, we can conclude that this regime is more effective than the others. There are two main conclusions that can be drawn from Figure 5. First, rather surprisingly, the linearity of the graph suggests that there is little difference among the vaccination regimes. Second, the cluster of points in the lower right corner of Figure 5 indicates the rate that people need to be vaccinated to eliminate cases and approach herd immunity: that is, approximately $0.027-0.028$ vaccinations per person per time. In other words, the figure suggests that approximately $2.7-2.8 \%$ of the population must be vaccinated every year in order to achieve herd immunity.

However, a closer look at these regimes reveals some important qualitative differences. In order to make fair comparisons, we examine four regimes that vaccinate the same average proportion of the population (see Table 3). These regimes (which come from the cluster of points in the lower right corner of Figure 5) seem to vaccinate at high enough rates to eliminate cases and thus move towards herd immunity.

However, there are qualitative differences in the number of cases across different types of cities (see Figure 6). Most notably, the dynamic infected regime exhibits substantially larger spikes in cases in the towns and regional centers. Since measles disappears for a while, these cities are vaccinating at low levels. However, as soon as an infected person enters the city, the number of cases spikes and an epidemic occurs. In contrast, the static constant regime does not result in these same kinds of spikes. There are a few outbreaks in the capital after cases have been more or less eliminated in other cities; however, this kind of behavior is to be expected, due to the fact that infected people from outside the country are immigrating relatively frequently to the capital. We added this rate to our model in order to ensure that measles does not completely disappear from the entire country. Note, however, that the towns and the regional centers do not experience any direct immigration from outside the country and still see spikes in cases. Although the static city regime does not result in the same 


\begin{tabular}{cccc}
\hline Regime & $\phi$ & Mean Prop. Vaccinated & Mean Prop. Infected \\
\hline \hline Static Constant & $\phi=0.06$ & 0.0281 & $1.541 \times 10^{-4}$ \\
Static City & $\phi_{T}=0.025, \phi_{R}=0.05, \phi_{C}=0.1$ & 0.0283 & $2.014 \times 10^{-4}$ \\
Dynamic Time & $\phi(t)=0.002 \sin \left(\frac{\pi}{13}(t+8)\right)+0.06$ & 0.0281 & $1.59 \times 10^{-4}$ \\
Dynamic Infected & $\phi=0.02 s+0.1 s 1_{\{i \neq 0\}}$ & 0.0281 & $2.521 \times 10^{-4}$ \\
\hline
\end{tabular}

Table 3: A comparison of the average proportions vaccinated and infected for four different regimes. Note the similarities in both the mean proportion vaccinated and the mean proportion infected. (Although the mean proportions infected look different, the differences are actually quite small-about 0.0001 or less. Notice, too, that these regimes fall in the lower right hand corner of Figure 5.)
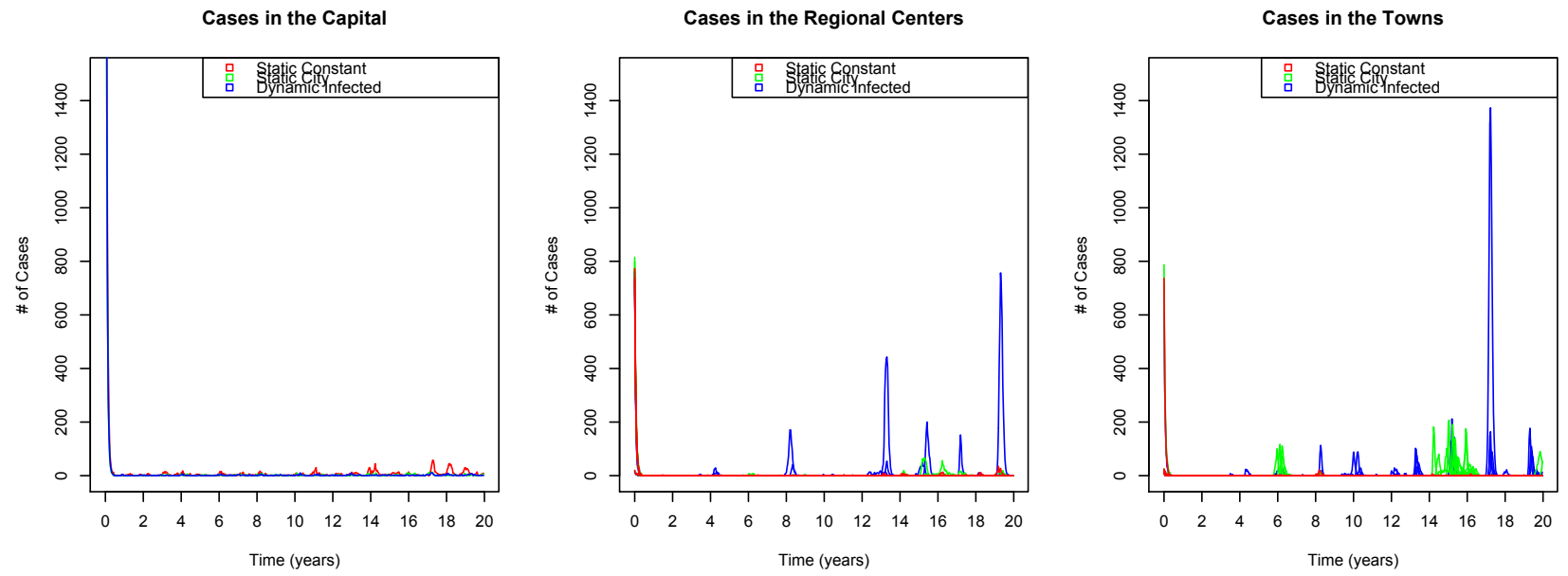

Figure 6: The number of cases for each of the three regimes from Table 3, with each type of city graphed in a different color. We omit the dynamic time regime because of its similarity to the static constant regime.

dramatic spikes as the dynamic infected regime, the towns do experience a few larger outbreaks for a similar reason - namely, a much lower vaccination rate in the towns than in the regional centers and the capital.

Because attaining herd immunity involves both eliminating cases and reducing the likelihood of future outbreaks, it is also important to consider the number of susceptibles in the population. To some extent, the proportion of susceptibles out of the total population suggests how stable the regime is. As the number of susceptibles increases, the population becomes more vulnerable to an outbreak. In a model with no vaccination, the proportion of susceptibles oscillates quite drastically (see Figure 3). This behavior accords with the behavior of cases. Since the number of cases oscillates, we expect the number of susceptibles to oscillate as well: the number of susceptibles decreases when people become infected and increases when measles disappears for a brief time. And of course, there are far higher proportions of susceptibles in a country without vaccination.

The differences in the proportions of susceptibles among the vaccination regimes are also illuminating (see Figure 7). The static city regime keeps the proportions of susceptibles in the capital and regional centers lowaround $1 \%$ and $2 \%$ respectively. However, the proportions of susceptibles in the towns are significantly higher, reaching about $5 \%$. Recall that the towns have much lower vaccination rates under this regime, which is based on the idea that concentrating vaccination in larger cities will control cases in the smaller cities. Thus, it is unsurprising that the towns have a large proportion of susceptibles. For a while, the towns remain measles-free since they are further away from the population centers; however, when an infected person happens to enter the city, susceptibles quickly contract measles. This regime, then, serves the regional centers and capital well, but leaves the towns vulnerable.

The dynamic infected regime exhibits even more dramatic fluctuations in the proportions of susceptibles than the static city regime does. It is worth mentioning that the proportion of susceptibles in the capital is kept under control, remaining at about $1 \%$ over the 20-year period. However, the proportions of susceptibles in the regional centers and the towns are erratic, spiking and dropping quickly. The proportions under this regime, reaching almost $5-6 \%$ at times, are higher than any other regime. This is due to the fact that the dynamic 
infected regime only vaccinates when there are infected people in the city. Thus, measles disappears from the regional centers and the towns for a while, but cases spike when an infected person enters the city. Examining the proportion of susceptibles reveals the instability of this regime, a fact that was not evident from the mean proportion infected.

While the static city and dynamic infected regimes serve some cities better than others, the static constant and dynamic time regimes stabilize the proportions of susceptibles. Both regimes keep the proportions of susceptibles at around $2 \%$ of the population. Given that these regimes also have the same average number of cases, the similarity in the proportions of susceptibles indicates that the regimes behave quite similarly.
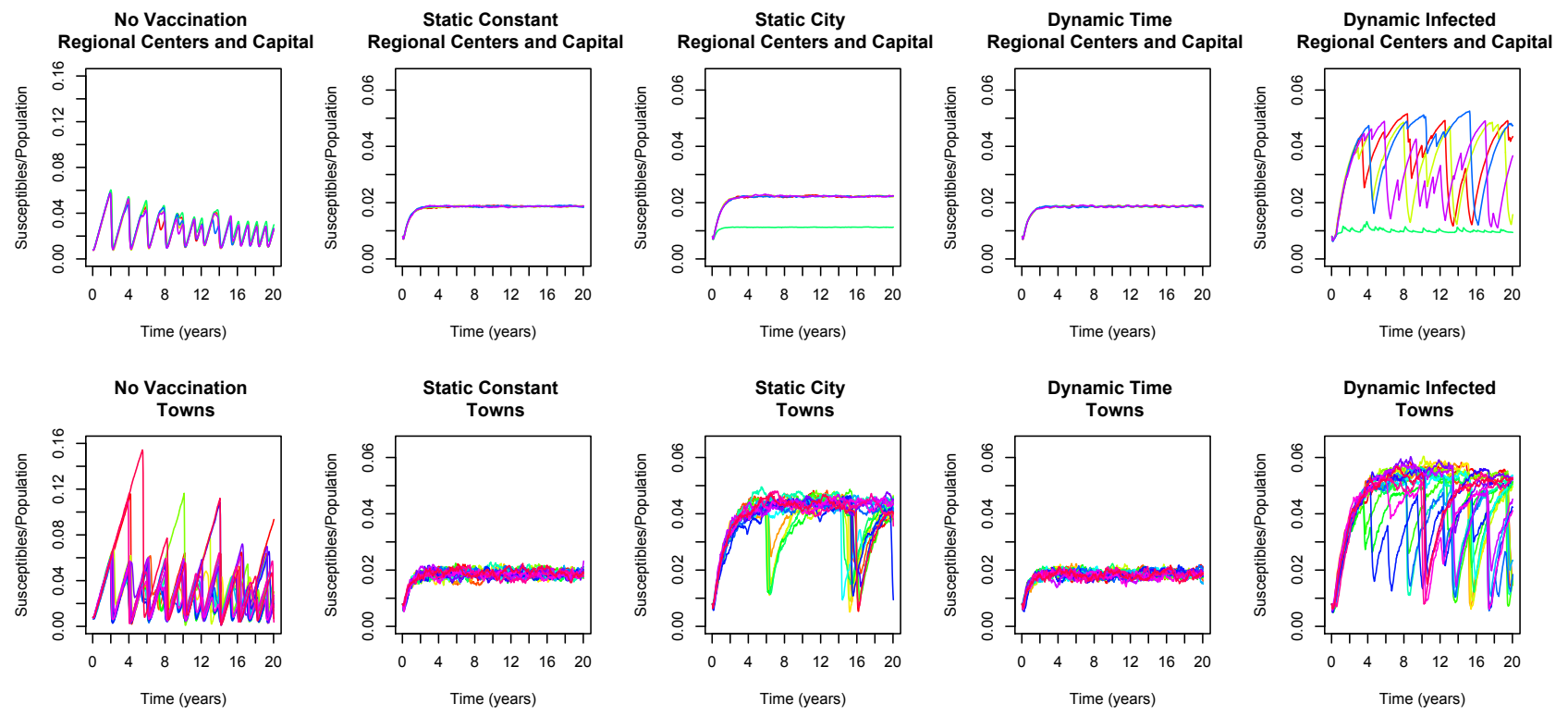

Figure 7: The proportion of susceptibles in the regimes from Table 3. We also include the proportion of susceptibles in a model with no vaccination for comparison (note the difference in scale on these graphs, which reach 0.16 on the y-axis, and the scale on the graphs of susceptibles under the vaccination regimes, which reach $0.065)$.

To summarize, the mean number of vaccinations and cases do not accurately reflect the qualitative behavior of the regimes as rates approach herd immunity. In the long term, the static constant and dynamic time regimes more effectively controlled cases than the other two regimes. The behavior of the dynamic infected regime, in particular, can be seen as a cautionary tale. To some extent, this regime mimics the behavior of a country in which measles has been mostly eliminated. As the disease starts to fade from the public consciousness and/or is no longer deemed a public health concern, fewer people are vaccinated. After a sufficient number of susceptibles has built up and an infected person enters the country, cases appear and people begin vaccinating quickly. Interestingly, this regime may arise from the behavior of the general population. Unfortunately, vaccinations do not happen quickly enough to prevent an epidemic. Our results suggest that lowering vaccination levels when measles seems to have been eliminated or stopping vaccinations altogether will lead to future epidemics. Based on these results, obtaining herd immunity involves not only eliminating cases but also maintaining a stable proportion of susceptibles; otherwise, measles has the opportunity to re-emerge with some virulence.

\subsection{Similarities between the Static Constant and Dynamic Time Regimes}

The similarity in the behavior of the static constant and dynamic time regimes seems to hold regardless of the level of vaccination. In particular, these regimes see the same proportion of susceptibles in the towns, and epidemics seem to occur at approximately the same time. Figure 8 demonstrates the similarity in the behavior of cases at different vaccination rates.

Although the number of cases is not always the same, the cases tend to spike periodically in both regimes; moreover, these spikes occur around the same time. The static constant and dynamic time regimes also work 
similarly in terms of controlling susceptibles in the towns (see Figure 9). There are frequent oscillations when vaccinating at low levels, but the proportion of susceptibles in the towns becomes more stable as the vaccination level increases.

The quantitative and qualitative similarities between these two regimes are somewhat surprising, since it seems logical that increasing vaccinations before the peak infection rate hits the country would help to control cases. This suggests that, to some extent, it matters little when people are vaccinated as long as they are vaccinated at some point during the year.
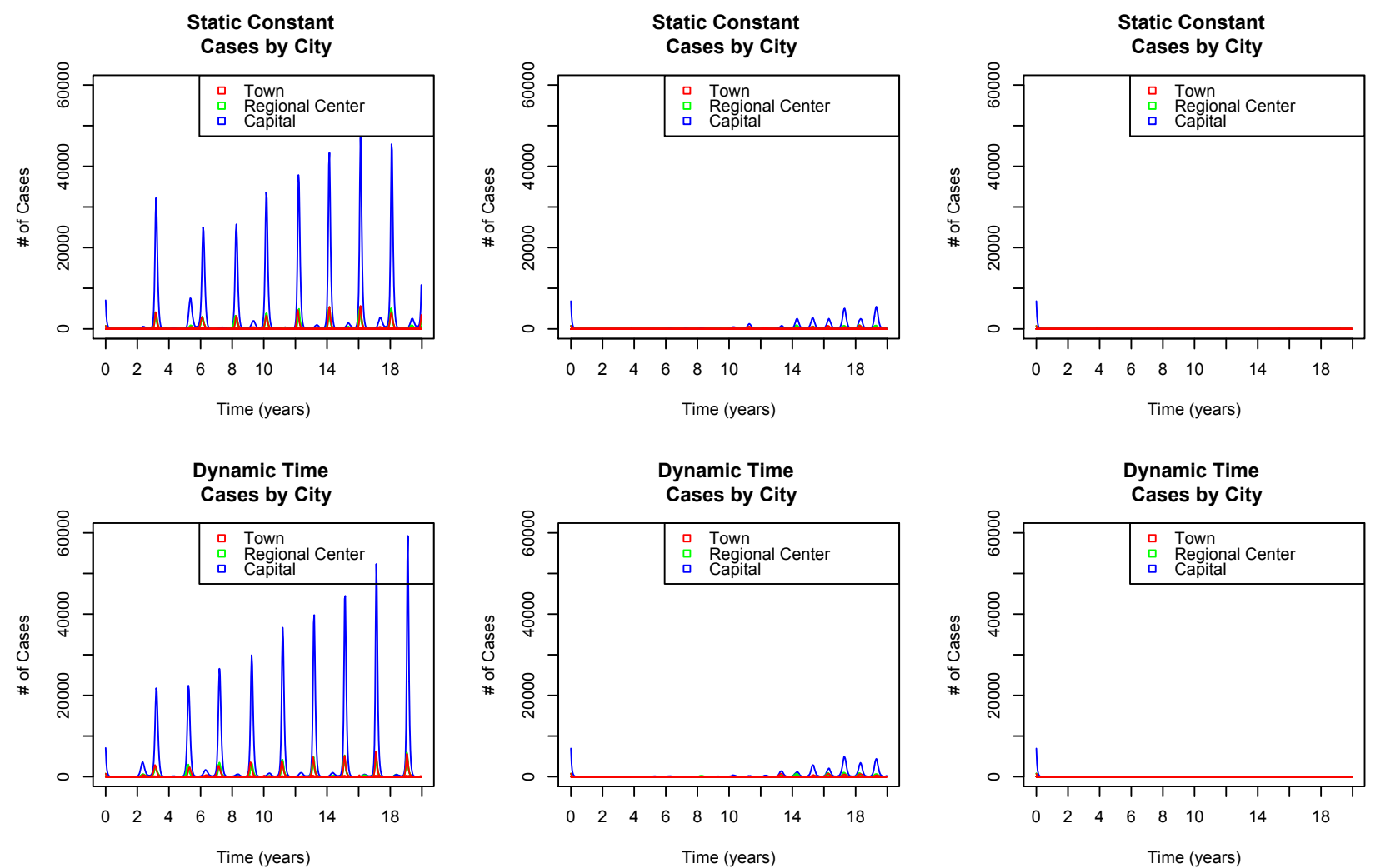

Figure 8: The number of cases by city under three different static constant regimes and dynamic time regimes. The regimes are grouped by the value of $\phi$ or the vertical shift $a$. That is, $\phi=a=0.02$ for the first column, $\phi=a=0.04$ for the second, and $\phi=a=0.06$ for the third.

\subsection{Static City Regime}

The static city regime was designed using a result from May and Anderson, who found that concentrating vaccination in the larger, denser city would eliminate cases while also requiring fewer vaccinations [May and Anderson, 1984].

\begin{tabular}{cccc}
\hline Regime & $\phi_{C}$ (capital) & $\phi_{R}$ (regional centers) & $\phi_{T}$ (towns) \\
\hline \hline 1 & 0.16 & 0 & 0 \\
2 & 0.16 & $\frac{1}{2} \phi_{C}=0.08$ & 0 \\
2 & 0.16 & $\frac{1}{2} \phi_{C}=0.08$ & $\frac{1}{4} \phi_{C}=0.04$ \\
\hline
\end{tabular}

Table 4: A table of three of the tests we ran for the static city regime. The capital is always vaccinated at a high rate.

Although May and Anderson's model was spatially similar to ours, our stochastic model did not produce the same result. Concentrating vaccination in the capital did not effectively eliminate measles from the entire country, let alone require fewer vaccinations to do so. This is clear from the behavior of three of the static city 

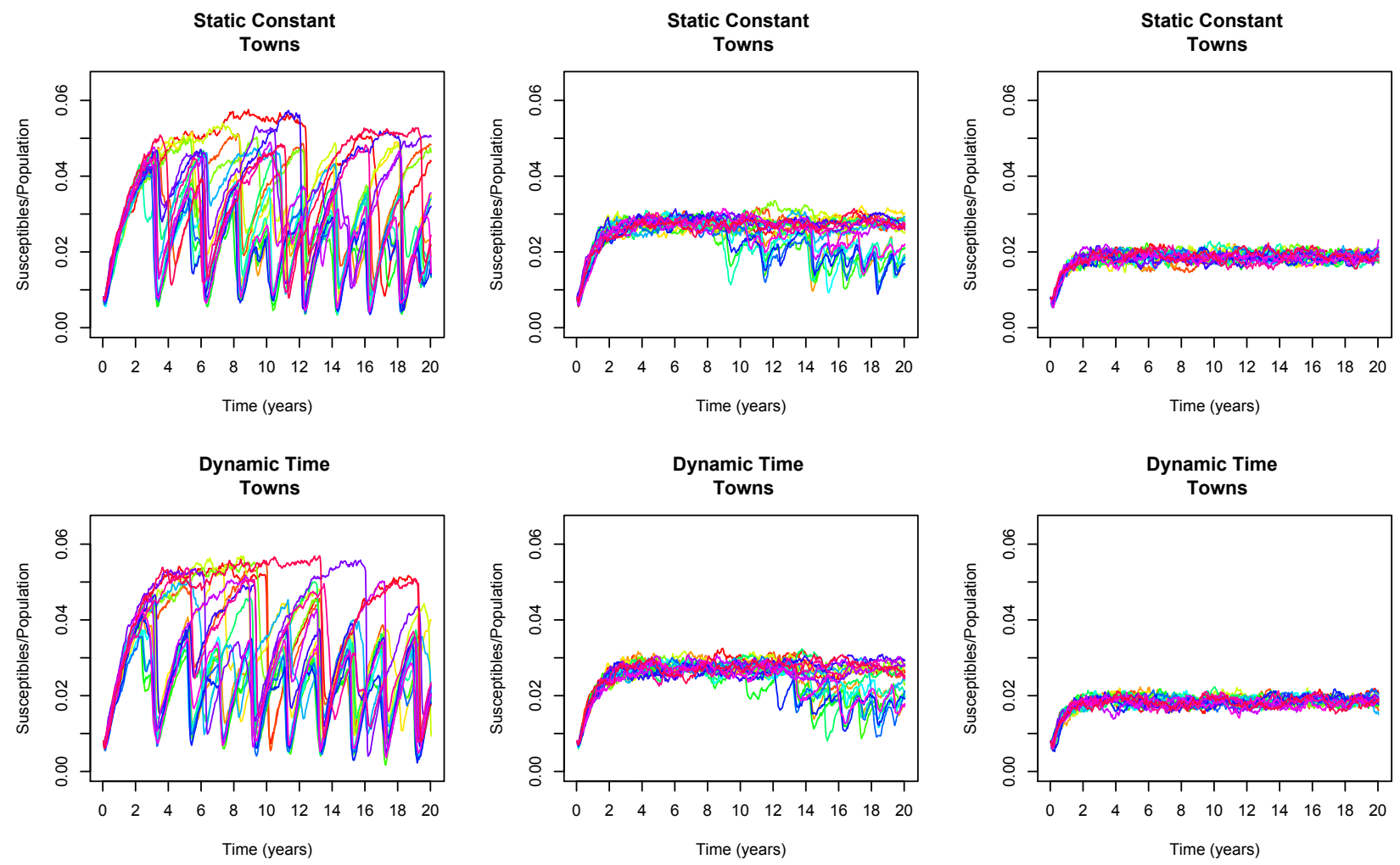

Figure 9: The proportion of susceptibles in the towns under three different static constant regimes and dynamic time regimes. The regimes are grouped by the value of $\phi$ or the vertical shift $a$. That is, $\phi=a=0.02$ for the first column, $\phi=a=0.04$ for the second, and $\phi=a=0.06$ for the third. Note the strong similarities between each set of graphs.

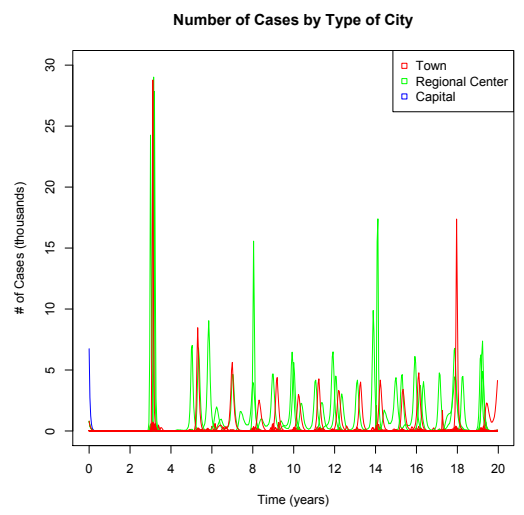

Figure 10: Vaccination only in the capital $\left(\phi_{C}=0.16, \phi_{R}=0\right.$, $\left.\phi_{T}=0\right)$.

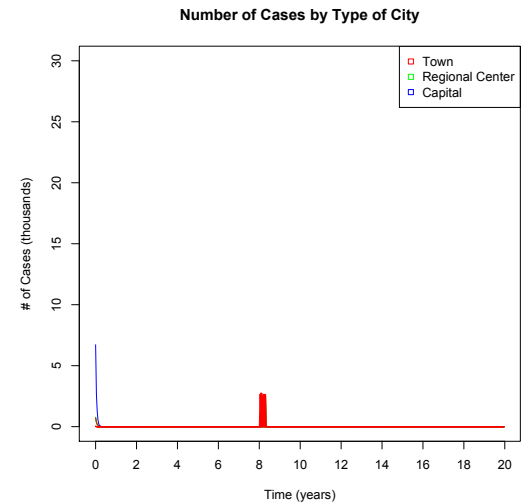

Figure 11: Vaccination concentrated in the capital with some vaccination in the regional centers $\left(\phi_{C}=0.16, \phi_{R}=0.08, \phi_{T}=0\right)$.

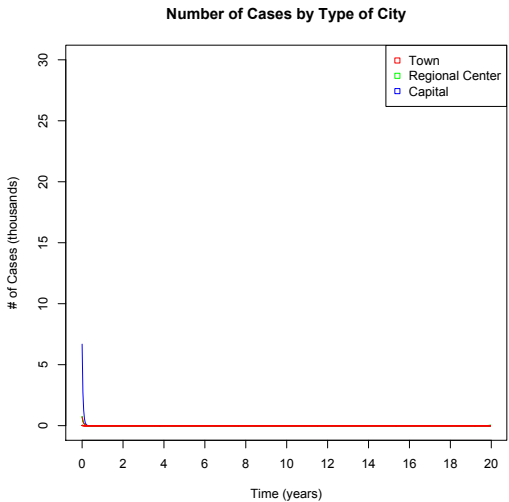

Figure 12: Vaccination in all cities, with higher levels in areas of higher population density $\left(\phi_{C}=\right.$ $\left.0.16, \phi_{R}=0.08, \phi_{T}=0.04\right)$.

regimes that we tested, listed in Table 4. When only the capital is vaccinated, in the first regime of Table 4, cases of course remain low in the capital. However, the regional centers and towns see large spikes after a brief period without cases (see Figure 10). We also examined a regime that includes a vaccination rate in the regional centers, which is half the rate in the capital. Under this regime, the towns still see a spike in cases after 


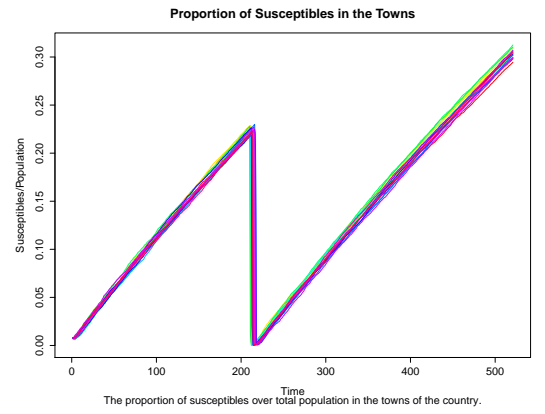

Figure 13: Proportion of town populations that are susceptible with vaccination concentrated in the capital and some vaccination in the regional centers $\left(\phi_{C}=0.16, \phi_{R}=\right.$ 0.08 , and $\left.\phi_{T}=0\right)$.

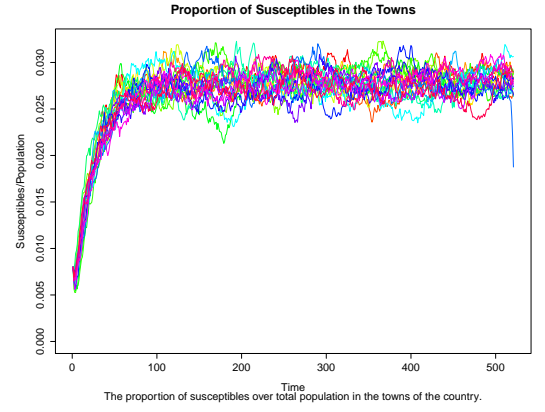

Figure 14: Proportion of town populations that are susceptible with vaccination in all cities $\left(\phi_{C}=0.16\right.$, $\phi_{R}=0.08$, and $\left.\phi_{T}=0.04.\right)$. (See Figure 12 for a graph of cases under this regime.)

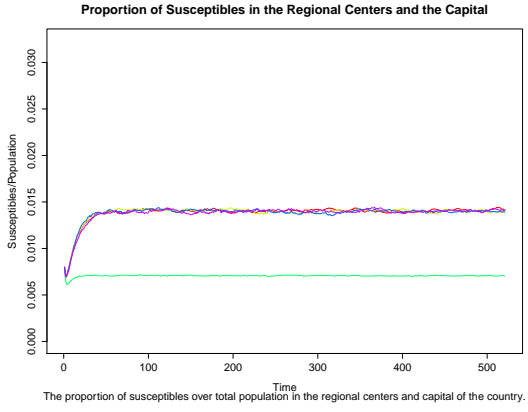

Figure 15: Proportion of regional center and capital populations that are susceptible with vaccination in all cities $\left(\phi_{C}=0.16, \phi_{R}=0.08\right.$, and $\phi_{T}=0.04$.). (See Figure 12 for a graph of cases under this regime.)

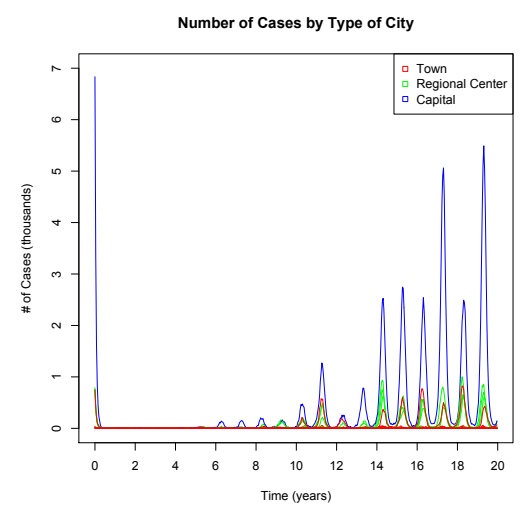

Figure 16: The number of cases in the static city regime at $\phi=0.04$. Note the spike in cases towards the end of the 20-year period.

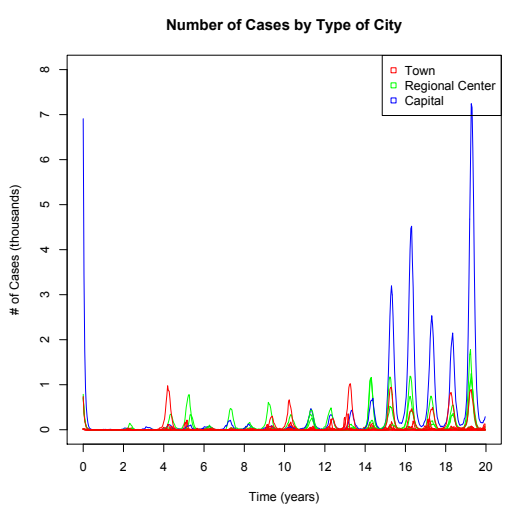

Figure 17: The number of cases in the dynamic infected regime at $\phi_{1}=0.02$ and $\phi_{2}=0.02$. Note the spikes in cases in the capital beginning around year 15, as well as the consistent outbreaks in the towns and regional centers.

a period without cases (see Figure 11). Moreover, the proportion of susceptibles in the towns is increasing at the end of the 20-year period, suggesting that there would be a substantial spike in cases if an infected person were to enter a town (see Figure 13).

Adding a vaccination rate to the towns seems to help control cases, as demonstrated by the third regime in Table 4 (see Figure 12). The regional centers and the capital fare quite well: the proportions of susceptibles stabilize at about $1.5 \%$ in the regional centers and well below $1 \%$ in the capital (see Figure 15). The proportions of susceptibles in the towns mostly stay below $3 \%$, which seems sufficient to keep cases from spiking (see Figure 14). While this regime seems to eliminate cases, the towns still have a significant number of susceptibles, which leaves them somewhat vulnerable to infected immigration. Thus, concentrating vaccination in the largest, densest city in the country does not seem to be an effective vaccination strategy in our model.

\subsection{Regimes at Lower Levels of Vaccination}

Although we have less data on regimes that vaccinate at lower levels, the data we do have suggest that we may have to modify our observations for these lower levels. A closer comparison of the dynamic infected regime at $0.02 s+0.02 s 1_{\{i \neq 0\}}$ and the static constant regime at $0.04 s$ is indicative. On average, this dynamic 
infected regime vaccinated approximately $2.58 \%$ of the total population every year, with the static constant regime slightly higher at $2.60 \%$. As we can see from Figures 16 and 17, all types of cities are still experiencing outbreaks at the end of the 20-year period. The most notable difference between these two regimes is that the static constant regime seems to more or less eliminate cases for about the first six years, while the dynamic infected regime still sees cases in the towns and regional centers.

Moreover, static constant regimes with $\phi<0.06$ still result in cases at the end of the 20-year period (as in Figure 16). Although cases may decrease at some point during the 20-year period, the fact that they return suggests that these regimes do not vaccinate at high enough levels to eliminate cases.

\section{Conclusion}

In addition to revealing the two most successful regimes in terms of attaining herd immunity, our simulations suggest the importance of examining the qualitative behavior of the vaccination regimes when evaluating their success. Moreover, differences in models with regard to stochastic versus deterministic methods, spatial structure, etc. may yield different results when the goal is to identify a suitable vaccination regime.

With regard to the four regimes we examined in depth, the dynamic infected and static city regimes still resulted in some measles cases towards the end of the 20-year period. However, the dynamic time and static constant regimes did well at both controlling the number of cases in the country and the proportion of susceptibles. If the goal is to reach herd immunity, the static constant and dynamic time regimes are the best choices out of the four regimes we examined.

We next observe that means do not necessarily reflect the qualitative behavior of the regimes. The qualitative differences we observed indicate the importance of considering other measures when determining the efficacy of a regime and whether it leads to herd immunity. In our work, we looked at the number of cases and proportion of susceptibles by city. Measures such as these indicate where cases are occurring, who is susceptible, and how vulnerable a particular city is. These measures seem to be especially important in spatial models, as different pockets of the population may experience different phenomena.

Finally, we reached a different conclusion than the May and Anderson results with regard to the best vaccination regime. There are, of course, important differences between our models. Most obviously, May and Anderson used a deterministic system of differential equations as opposed to stochastic simulations to create their model. In addition, although both models were spatial, May and Anderson had only one big city with smaller cities, while we had three classes of cities (towns, regional centers, and the capital). Despite these differences, it is possible that adding randomness to the model makes it difficult to control the number of cases by only vaccinating the big city. This is certainly worthy of future consideration.

There are some other interesting avenues for future research. First and foremost, it would be interesting to consider age dynamics in our model, as measles generally affects younger children more frequently than adults. It would also be worth considering other immigration and population concentration patterns. We only allow horizontal and vertical emigration in our model for the sake of simplicity; however, this is not necessarily realistic, as it is now equally easy for people to make diagonal movements around many countries. Moreover, the spatial structure of our country may not accurately reflect the structure of modern countries. Because transportation has become much more rapid, people may travel from the capital to the towns and vice versa more frequently. Finally, most of the tests we ran on our vaccination regimes resulted in higher levels of vaccination. It would be interesting to collect more data on regimes at lower levels so that we could make better comparisons and identify any significant qualitative differences when high levels of vaccination are not possible.

\section{Acknowledgements}

We would like to thank the Mathematical Biosciences Institute for supporting our research and the NSF for funding our project. A special thank you goes to our advisor, Dr. Fricks, for his constant support throughout this entire project.

\section{Appendix A Numerical Methods}

There are two methods that can be used to simulate the number of people in each compartment, one of which is exact and computationally expensive and one of which gives an approximation and is computationally expedient. 
In this section, as in $\S 2.1$, we describe the methods for simulating the model for one city for simplicity. However, the final model works very similarly.

\section{A.0.1 Exact Method: Gillespie SSA}

The exact method uses the Gillespie SSA to calculate the values of the compartments through time. This method simulates the time between events and then determines which event occurs at that time [Gillespie, 1977]. $\tau$ represents the time between the event that has just occurred and the next event. To determine $\tau$, the model simulates an exponential random variable with the sum of the rates as the probability of the exponential distribution. In one city, recall that we have the following rates at time $t$ (excluding emigration): $\mu_{B} N(t)$, $\beta S(t) I(t), \phi S(t), \omega I(t)$, and $\mu_{D} N(t)$. Thus the mean of the exponential distribution is

$$
\lambda=\mu_{B} N(t)+\beta S(t) I(t)+\phi S(t)+\omega I(t)+\mu_{D} N(t) .
$$

In order to determine which event happens at the new time, we first calculate the probability that each event occurs. This is given by the rate of the event over the sum of all rates. For example, the probability that a birth occurs is

$$
\frac{\mu_{B} N(t)}{\mu_{B} N(t)+\beta S(t) I(t)+\phi S(t)+\omega I(t)+\mu_{D} N(t)} .
$$

We can collect all of these probabilities in a vector:

$$
\left(\begin{array}{c}
\frac{\mu_{B} N(t)}{\frac{\beta(t)+\phi S(t)+\omega I(t)+\mu_{D} N(t)}{\mu_{B} N(t)+\beta S(t) I(t)+(t)}} \\
\frac{\beta S(t) I(t)+\omega I(t)+\mu_{D} N(t)}{\mu_{B} N(t)+\beta S(t) I(t)+\phi S(t)+\omega I(t)+\mu^{\prime}} \\
\frac{\phi S(t)}{\mu_{B} N(t)+\beta S(t) I(t)+\phi S(t)+\omega I(t)+\mu_{D} N(t)} \\
\frac{\omega I(t)}{\mu_{B} N(t)+\beta S(t) I(t)+\phi S(t)+\omega I(t)+\mu_{D} N(t)} \\
\overline{\mu_{B} N(t)+\beta S(t) I(t)+\phi S(t)+\omega I(t)+\mu_{D} N(t)}
\end{array}\right) .
$$

Using these probabilities, we simulate an integer that corresponds to a particular reaction (a birth, death, infection, recovery, or vaccination) and thus tells us which event has occurred.

Finally, we must adjust the values of the compartments based on the reaction that has just occurred. The changes to the compartments are specified by the column of $A$ that corresponds to the reaction that has occurred. For example, if a birth occurred, we add 1 to S. If an infection occurs, we subtract 1 from $\mathrm{S}$ and add 1 to I.

This process occurs many times until the terminal time, $T$, is reached. If $\tau_{i}$ is the time between event $i-1$ and event $i$, then we call $\eta_{i}$ the time at which event $i$ actually occurs. We can easily calculate $\eta_{i}$ by summing $\tau_{1}, \tau_{2}, \ldots, \tau_{i}$. Thus, we can also say that the process ends when $\eta_{i} \geq T$.

\section{A.0.2 Approximate Method}

The exact simulation technique only estimates one event at a time. However, we can also use an approximate method called "tau-leaping," which has a higher accuracy when working with large numbers [Anderson et al., 2011, Rathinam et al., 2003, Rock et al., 2014]. This method hinges on estimating the number of events that occur over a given time interval. First, an "appropriate" time step, $\Delta$, must be chosen. Each event in the model (births, deaths, infections, vaccinations, and recoveries) can then be simulated by a Poisson random variable, where the mean of the distribution is the rate of the event scaled by the time step. The outcome of each Poisson random variable represents the number of times that each event has occurred in time step $\Delta$. The approximation works because the chosen $\Delta$ is sufficiently small - that is, the rates do not vary over that time step. The process continues until the terminal time, $T$, is reached. For example, if $\Delta=0.01$ and $T=1$, the model approximates the value of each compartment at $t=0.01,0.02,0.03,0.04,0.05, \ldots, 1$.

In short, we do an Euler approximation of the integrals in the time-changed unit one Poisson processes in (1). The approximation of the compartments at time $t+\Delta$ works as follows:

$$
\left(\begin{array}{c}
S(t+\Delta) \\
I(t+\Delta) \\
R(t+\Delta)
\end{array}\right)=\left(\begin{array}{c}
S(t) \\
I(t) \\
R(t)
\end{array}\right)+A\left(\begin{array}{c}
Y_{1}\left(\int_{t}^{t+\Delta} \mu_{B} N(s) d s\right) \\
Y_{2}\left(\int_{t}^{t+\Delta} \beta S(s) I(s) d s\right) \\
Y_{3}\left(\int_{t}^{t+\Delta} \phi S(s) d s\right) \\
Y_{4}\left(\int_{t}^{t+\Delta} \omega I(s) d s\right) \\
Y_{5}\left(\int_{t}^{t+\Delta} \mu_{D} N(s) d s\right)
\end{array}\right) \approx\left(\begin{array}{c}
S(t) \\
I(t) \\
R(t)
\end{array}\right)+A\left(\begin{array}{c}
Y_{1}^{*}\left(\mu_{B} N(t) \Delta\right) \\
Y_{2}^{*}(\beta S(t) I(t) \Delta) \\
Y_{3}^{*}(\phi S(t) \Delta) \\
Y_{4}^{*}(\omega I(t) \Delta) \\
Y_{5}^{*}\left(\mu_{D} N(t) \Delta\right)
\end{array}\right)
$$


So far, we have looked at the methods for calculating the values of the compartments of one city. However, our final model has 75 total compartments (three for each of the 25 cities) and 206 reactions. Due to the size of the model, the exact method takes a long time to run. Approximately 60,000 events occur within a two-week period; run the model for 20 years, and that number enters the tens of millions. After making sure that the exact and approximate methods give similar results, we opted to use the approximate method at $\Delta=0.001$. At this time scale, we are collecting the number of events that occur about every 20.16 minutes.

\section{Appendix B Parameters}

This appendix describes how and why the values for the parameters in Table 1 were chosen. As mentioned before, most of the parameter values are based on the analysis performed by Bjørnstad et al. on pre-vaccination era data from England and Wales. We will discuss the parameters for five of the events that may occur in our model - that is, births, deaths, recoveries, infections, and emigrations. Note that vaccination parameters are discussed in $\S 3$ and are specific to each regime.

The birth rate $\left(\mu_{B}\right)$, death rate $\left(\mu_{D}\right)$, and recovery rate $(\omega)$ remain constant across the population and across time - that is, there is one birth rate, one death rate, and one recovery rate for the entire country. After World War II, the annual per capita birth rate in Britain was 0.01-0.02 [Bjørnstad et al., 2002]. We increase this rate to 0.03 to achieve a $2-3 \%$ increase in population every year. Thus,

$$
\mu_{B}=\log (1.03)\left(\frac{1}{26}\right) \approx 0.001136877 .
$$

Since we are using time units of two weeks, we divide $\log (1.03)$ by 26 to change the units from one year to two weeks. We use log because 1.03 is compounded. In order to ensure that the population grows by about $2-3 \%$ every year, we set the death rate at

$$
\mu_{D}=0.00025 .
$$

Finally, the recovery rate is the inverse of the infectious period [Allen et al., 2008]. The average infectious period is 14 days [Bjørnstad et al., 2002]. After changing the unit of time to two weeks, we have

$$
\omega=\frac{1}{14 \text { days }}=\frac{1}{1 \text { two-week period }}=1 .
$$

As mentioned in $\S 2.2$, the infection rates are seasonal. Because of the way these seasonal infection rates are constructed, each type of city has a different infection rate. Thus we have three infection rates, each a function of time: $\beta_{T}(t), \beta_{R}(t)$, and $\beta_{C}(t)$. We model $\beta_{*}(t)$ after the graph of the seasonal deviation in $\log \beta$ from Bjørnstad et al.'s paper [Bjørnstad et al., 2002] (Fig. 7 B on p. 178):

$$
\begin{aligned}
\beta_{t} & =\bar{\beta}-0.4 \bar{\beta} \sin \left(\frac{2 \pi}{26}(t+\theta)\right) \\
& =\bar{\beta}-0.4 \bar{\beta} \sin \left(\frac{\pi}{13}(t+\theta)\right) .
\end{aligned}
$$

In order to calculate $\bar{\beta}$, we use $\log (\bar{\beta})=3.64-1.02 \log (N)$ from Bjørnstad et al. and exponentiate [Bjørnstad et al., 2002]. It is for this reason that the infection rate depends on the size of the city, and we use the initial population of each city $\left(N_{T}=10,000, N_{R}=500,000\right.$, and $\left.N_{C}=5,000,000\right)$ to calculate $\bar{\beta}$.

\begin{tabular}{cccc}
\hline Type of City & $\log (\beta)$ & approximate & $\beta$ \\
\hline \hline Towns & $3.64-1.02 \log (10,000)$ & -5.775 & 0.0031 \\
Regional Centers & $3.64-1.02 \log (500,000)$ & -9.745 & 0.000059 \\
Capital & $3.64-1.02 \log (5,000,000)$ & -12.093 & 0.0000056 \\
\hline
\end{tabular}

Table 5: A table of the values for $\log (\bar{\beta})$ and $\beta$ for each type of city. 
We use the same graph given in the Bjørnstad paper (see Fig. 7 (B) on p. 178) [Bjørnstad et al., 2002] to find $\theta$. The sine function is shifted so that $\beta$ is lowest in August; that is, $\theta=16.5$. We can now approximate $\beta_{*}(t)$ for each city:

- $\beta_{T}(t)=0.0031-2.3018 \bar{\beta} \sin \left(\frac{\pi}{13}(t+16.5)\right)$,

- $\beta_{R}(t)=0.000059-3.8979 \bar{\beta} \sin \left(\frac{\pi}{13}(t+16.5)\right)$, and

- $\beta_{C}(t)=0.0000056-4.8374 \bar{\beta} \sin \left(\frac{\pi}{13}(t+16.5)\right)$.

The last parameters that remain to be discussed are the emigration rates. Each type of city has a different emigration rate, a decision that is again grounded in Bjørnstad et al.'s analysis of pre-vaccination era data in England and Wales. According to Bjørnstad et al., the immigration rate "scales roughly with the square root of the population size" [Bjørnstad et al., 2002]. This translates to larger emigration rates for larger cities. The paper has estimated values for $m$ for populations of 10,000, 111,000,300,000, and 764,000 on page 177. $m$ varies widely for the city of 764,000, ranging from 0.2 to 20 [Bjørnstad et al., 2002]. Values for $m$ were chosen from that range, and as with infection rates, we used the initial population size of each city to calculate $\delta_{T}, \delta_{R}$, and $\delta_{C}($ Table 6$)$.

\begin{tabular}{ccc}
\hline Type of City & $m$ & $\delta$ \\
\hline \hline Capital & 20 & $\frac{20}{\sqrt{5,000,000}} \approx 0.008944272\left(\delta_{C}\right)$ \\
Regional Centers & 4 & $\frac{4}{\sqrt{500,000}} \approx 0.005656854\left(\delta_{R}\right)$ \\
Towns & 0.2 & $\frac{.2}{\sqrt{10,000}}=0.002\left(\delta_{T}\right)$ \\
\hline
\end{tabular}

Table 6: A table of the values for $m$ chosen for each type of city and the resulting emigration rate.

\section{References}

[Allen, 2010] Allen, L. J. (2010). An introduction to stochastic processes with applications to biology. CRC Press.

[Allen et al., 2008] Allen, L. J., Brauer, F., Van den Driessche, P., and Wu, J. (2008). Mathematical epidemiology. Springer.

[Anderson et al., 2011] Anderson, D. F., Ganguly, A., and Kurtz, T. G. (2011). Error analysis of tau-leap simulation methods. The Annals of Applied Probability, pages 2226-2262.

[Anderson and May, 1991] Anderson, R. M. and May, R. M. M. (1991). Infectious diseases of humans: dynamics and control. Oxford University Press.

[Ball and Neal, 2002] Ball, F. and Neal, P. (2002). A general model for stochastic sir epidemics with two levels of mixing. Mathematical biosciences, 180(1):73-102.

[Ball and Lyne, 2002] Ball, F. G. and Lyne, O. D. (2002). Optimal vaccination policies for stochastic epidemics among a population of households. Mathematical biosciences, 177:333-354.

[Bjørnstad et al., 2002] Bjørnstad, O. N., Finkenstädt, B. F., and Grenfell, B. T. (2002). Dynamics of measles epidemics: estimating scaling of transmission rates using a time series sir model. Ecological Monographs, $72(2): 169-184$.

[Bolker and Grenfell, 1995] Bolker, B. and Grenfell, B. (1995). Space, persistence and dynamics of measles epidemics. Philosophical Transactions of the Royal Society of London B: Biological Sciences, 348(1325):309320.

[Britton, 2010] Britton, T. (2010). Stochastic epidemic models: a survey. Mathematical biosciences, 225(1):2435. 
[Calandrillo, 2004] Calandrillo, S. (2004). Vanishing vaccinations: why are so many americans opting out of vaccinating their children? University of Michigan Journal of Law Reform, 37:353.

[Caudron et al., 2015] Caudron, Q., Mahmud, A., Metcalf, C., Gottfreðsson, M., Viboud, C., Cliff, A., and Grenfell, B. (2015). Predictability in a highly stochastic system final size of measles epidemics in small populations. Journal of The Royal Society Interface, 12(102):20141125.

[CDC, 2015a] CDC (2015a). Measles Vaccination. http://www.cdc.gov/measles/vaccination.html. [Online; accessed July 14, 2015].

[CDC, 2015b] CDC (2015b). Vaccines Do Not Cause Autism. http://www.cdc.gov/vaccinesafety/ concerns/autism.html. [Online; accessed January 7, 2016].

[Dalziel et al., 2016] Dalziel, B. D., Bjørnstad, O. N., van Panhuis, W. G., Burke, D. S., Metcalf, C. J. E., and Grenfell, B. T. (2016). Persistent chaos of measles epidemics in the prevaccination united states caused by a small change in seasonal transmission patterns. PLoS Comput Biol, 12(2):e1004655.

[Ferrari et al., 2013] Ferrari, M., Grenfell, B., and Strebel, P. (2013). Think globally, act locally: the role of local demographics and vaccination coverage in the dynamic response of measles infection to control. Phil. Trans. R. Soc. B, 368(1623):20120141.

[Fine, 1993] Fine, P. E. (1993). Herd immunity: history, theory, practice. Epidemiologic reviews, 15(2):265-302.

[Flaherty, 2011] Flaherty, D. K. (2011). The vaccine-autism connection: a public health crisis caused by unethical medical practices and fraudulent science. Annals of Pharmacotherapy, 45(10):1302-1304.

[Freymuth and Vabret, 2011] Freymuth, F. and Vabret, A. (2011). Measles, a re-emerging disease in france? Clinical Microbiology and Infection, 17(6):793-793.

[Gillespie, 1977] Gillespie, D. T. (1977). Exact stochastic simulation of coupled chemical reactions. The journal of physical chemistry, 81(25):2340-2361.

[Grammens et al., 2016] Grammens, T., Maes, V., Hutse, V., Laisnez, V., Schirvel, C., Trémérie, J. M., and Sabbe, M. (2016). Different measles outbreaks in belgium, january to june 2016-a challenge for public health. Eurosurveillance, 21(32).

[Grenfell et al., 2001] Grenfell, B., Bjørnstad, O., and Kappey, J. (2001). Travelling waves and spatial hierarchies in measles epidemics. Nature, 414(6865):716-723.

[Kidd et al., 2012] Kidd, S., Ouedraogo, B., Kambire, C., Kambou, J. L., McLean, H., Kutty, P. K., Ndiaye, S., Fall, A., Alleman, M., Wannemuehler, K., et al. (2012). Measles outbreak in burkina faso, 2009: a case-control study to determine risk factors and estimate vaccine effectiveness. Vaccine, 30(33):5000-5008.

[Majumder et al., 2015] Majumder, M. S., Cohn, E. L., Mekaru, S. R., Huston, J. E., and Brownstein, J. S. (2015). Substandard vaccination compliance and the 2015 measles outbreak. JAMA pediatrics, 169(5):494495.

[May and Anderson, 1984] May, R. M. and Anderson, R. M. (1984). Spatial heterogeneity and the design of immunization programs. Mathematical Biosciences, 72(1):83-111.

[Olsen and Schaffer, 1990] Olsen, L. F. and Schaffer, W. M. (1990). Chaos versus noisy periodicity: alternative hypotheses for childhood epidemics. Science, 249(4968):499-504.

[Rathinam et al., 2003] Rathinam, M., Petzold, L. R., Cao, Y., and Gillespie, D. T. (2003). Stiffness in stochastic chemically reacting systems: The implicit tau-leaping method. The Journal of Chemical Physics, 119(24):12784-12794.

[Rock et al., 2014] Rock, K., Brand, S., Moir, J., and Keeling, M. J. (2014). Dynamics of infectious diseases. Reports on Progress in Physics, 77(2):026602.

[Verdasca et al., 2005] Verdasca, J., Da Gama, M. T., Nunes, A., Bernardino, N., Pacheco, J., and Gomes, M. (2005). Recurrent epidemics in small world networks. Journal of Theoretical Biology, 233(4):553-561. 
[WHO, 2015] WHO (2015). Measles. http://www.who.int/mediacentre/factsheets/fs286/en/. [Online; accessed July 13, 2015].

[Zipprich et al., 2015] Zipprich, J., Winter, K., Hacker, J., Zia, D., Watt, J., and Harriman, K. (2015). Measles outbreak - california, december 2014-february 2015. Morbidity and Mortality Weekly Report, 64(6):153-154. 